University of Nebraska - Lincoln

DigitalCommons@University of Nebraska - Lincoln

Biochemistry -- Faculty Publications

Biochemistry, Department of

$2-1-2007$

\title{
Purification method directly influences effectiveness of an epidermal growth factor-coupled targeting agent for noninvasive tumor detection in mice
}

Joy L. Kovar

LI-COR Biosciences, joy.kovar@licor.com

William M. Volcheck

LI-COR Biosciences

Jiyan Chen

LI-COR Biosciences

Melanie A. Simpson

University of Nebraska - Lincoln, msimpson2@unl.edu

Follow this and additional works at: https://digitalcommons.unl.edu/biochemfacpub

Part of the Biochemistry, Biophysics, and Structural Biology Commons

Kovar, Joy L.; Volcheck, William M.; Chen, Jiyan; and Simpson, Melanie A., "Purification method directly influences effectiveness of an epidermal growth factor-coupled targeting agent for noninvasive tumor detection in mice" (2007). Biochemistry -- Faculty Publications. 10.

https://digitalcommons.unl.edu/biochemfacpub/10

This Article is brought to you for free and open access by the Biochemistry, Department of at DigitalCommons@University of Nebraska - Lincoln. It has been accepted for inclusion in Biochemistry -- Faculty Publications by an authorized administrator of DigitalCommons@University of Nebraska - Lincoln. 
Published in Analytical Biochemistry 361:1 (February 1, 2007), pp. 47-54; doi:10.1016/j.ab.2006.11.021 Copyright (C) 2006 Elsevier Inc. Used by permission. http://www.sciencedirect.com/science/journal/00032697

\title{
Purification method directly influences effectiveness of an epidermal growth factor-coupled targeting agent for noninvasive tumor detection in mice
}

\author{
Joy L. Kovar ${ }^{\text {, }}$ William M. Volcheck ${ }^{\text {, }}$, Jiyan Chen ${ }^{\text {, }}$, and Melanie A. Simpson ${ }^{\text {,** }}$ \\ ${ }^{a}$ LI-COR Biosciences, Lincoln, NE 68504, USA \\ b Department of Biochemistry, University of Nebraska, Lincoln, NE 68588, USA \\ *Corresponding author. Fax: 402 472-7842; Email: msimpson2@unl.edu (M. A. Simpson).
}

\begin{abstract}
Receptor targeting is an effective method of enhancing fluorescence signal in tumors for optical imaging. We previously used epidermal growth factor (EGF) conjugated to IRDye $800 \mathrm{CW}$ to detect and track orthotopic prostate tumors in mice. In this study, our goal was to identify a reliable assay for targeting agent integrity in vitro that correlated with signal strength in vivo. Binding of IRDye $800 \mathrm{CW}$ EGF to intact A431 human epidermoid carcinoma cells was quantified in a microplate assay. Specificity was confirmed by competition with unlabeled EGF or monoclonal antibody blocking. Biological activity of intact and damaged targeting agents relative to unlabeled EGF was determined by binding and stimulation of extracellular signal-regulated kinase (ERK) phosphorylation. Both assays indicated a reduction of up to $60 \%$ of the fluorescence intensity with damaged agents. Using a research prototype imaging system optimized for IRDye $800 \mathrm{CW}$ detection, we compared the efficacy of intact and damaged targeting agents for imaging subcutaneous tumors in mice. In live animal images and in sections of the excised tumors, damaged targeting agents consistently yielded diminished fluorescence signals corresponding to the reduction observed in microplate assays. This is the first study to directly correlate targeting agent signal strength in whole cell binding, In-Cell Western, and in vivo near-infrared imaging.
\end{abstract}

Keywords: EGF receptor, IRDye 800CW EGF, In-Cell Western assay, Binding affinity, Noninvasive, Whole animal imaging

Abbreviations: NIR, near-infrared; RGD, Arg-Gly-Asp; EGF, epidermal growth factor; ERK, extracellular signal-regulated kinase; ATCC, American Type Culture Collection; DMEM, Dulbecco's modified Eagle's medium; MEM, minimal essential medium; PBS, phosphate-buffered saline; FPLC, fast protein liquid chromatography; TEAA, triethylammonium acetate; CCD, charge-coupled device; SNR, signal-to-noise ratio; ROI, region of interest; FITC, fluorescein isothiocyanate.

Studies of tumor growth and metastasis in small animal models have benefited greatly from the expanded availability of optical imaging techniques that specifically detect tumor tissue in live animals [1] and [2]. A major advantage of optical imaging is the cost-effective accessibility of the requisite instrumentation. Several promising methods use molecular approaches to enhance the specificity of optical detection. Frequently, chosen techniques include measurement of bioluminescence, in which tumor cell lines bearing a luciferase reporter gene are detected by systemic delivery of a luminescent substrate, and gene-encoded fluorescence of tumor cell lines expressing green or red fluorescent proteins [3] and [4]. Although both tumor detection methods are powerful and sensitive, their major limitation is a requirement for specific cell lines. An alternative approach is the exploitation of high-affinity receptor-ligand interactions to target molecular features of the tumor cell with coupled fluorophores.

Targeting agents that have been used successfully for optical noninvasive detection of tumor xenografts in mice include monoclonal antibodies [5] and [6], small polypeptide growth factors [7] and [8], and peptide analogues of extracellular ligands such as endostatin [9] and somatostatin [10]. The most effective coupled fluorophores are those with fluorescence emission maxima in the near-infrared (NIR) range 
Kovar, Volcheck, Chen, \& Simpson in Analytical Biochemistry 361 (2007)

of 780 to $850 \mathrm{~nm}$. Due to low-absorbance spectra of bodily fluids and tissues, imaging in the NIR offers the advantages of relatively deep tissue penetration and low autofluorescence [11] and [12]. Examples include targeting of the integrin $\alpha_{v} \beta_{3}$ using variations of Arg-Gly-Asp (RGD) peptides linked to Cy5.5 [13], [14], [15], and [16], Alexa Fluor 680 [13], and IRDye 800CW [16]. Recently, epidermal growth factor (EGF) labeled with Cy5.5 was used to quantify therapeutic efficacy of anti-EGF receptor antibodies for treating mammary carcinoma in mice [8].

To optimize receptor-targeted signal strength in NIR optical imaging, a detailed understanding of the targeting mechanism and fluorophore function is critical. The EGF receptor is a potential biomarker for numerous preclinical models of human cancer because its expression is elevated an order of magnitude or more relative to normal cells in cancers of the breast [17], prostate [18], lung [19], ovary [20], and brain [21]. Furthermore, its signaling functions are well characterized and accessible to convenient high-throughput in vitro validation assays to ensure that bioactivity of the ligand is maintained despite chemical coupling. For example, EGF binds to a cell surface transmembrane receptor tyrosine kinase, and this induces dimerization of the receptors, triggering a phosphorylation cascade through numerous extracellular-regulated kinases [22]. Because antibodies are available to specifically quantify the phosphorylated form of several kinases, activity of EGF may be verified directly by receptor binding and indirectly by stimulated kinase activity.

We previously conjugated IRDye $800 \mathrm{CW}$ to EGF to track longitudinal growth of orthotopic prostate tumors in mice [23] and found that the conjugate was a sensitive tumor targeting agent with excellent tissue penetration. We hypothesized that an aspect of the normal function of EGF contributing to the strong signal obtained from this agent could be receptor-mediated endocytosis of the ligand-receptor complex. This would imply that initial high-affinity binding of the targeting agent is essential for maximal fluorescence signal strength in animals. Thus, animal numbers could be minimized in imaging experiments if suboptimal conjugate binding in vitro was found to correlate with reduced tumor targeting efficacy in vivo. Furthermore, it would be very powerful to have a straightforward in vitro assay that could directly predict relative efficiencies of two different drugs or ligands with the same in vivo target. In this study, we used IRDye $800 \mathrm{CW}$ EGF to target A431 human epidermoid carcinoma cells in vitro and in vivo. Specifically, we compared whole cell binding, extracellular signal-regulated kinase (ERK) phosphorylation, and subcutaneous tumor targeting of optimal and damaged conjugates. Our characterization confirms that poor quality of a receptor targeting agent can be detected by measuring in vitro binding equilibria and that reduced bioactivity is detrimental to imaging sensitivity.

\section{Materials and methods}

\section{Cell culture, materials, and reagents}

A431 human epithelial squamous carcinoma cells were purchased from American Type Culture Collection (ATCC, Manassas, VA, USA) and maintained in Dulbecco's modified Eagle's medium (DMEM) supplemented with $10 \%$ fetal bovine serum. PC3M-LN4 human prostate adenocarcinoma cells were kindly provided by Isaiah J. Fidler (M.D. Anderson Cancer Center, Houston, TX, USA) and maintained in minimal essential medium (MEM) containing 10\% fetal bovine serum, sodium pyruvate, and nonessential amino acids. A purified mouse diet (AIN-93M) was obtained from Harlan Teklad (Madison, WI, USA). Monoclonal antibody C225 (Cetuximab, ImClone Systems) was graciously provided by Chun Li (M. D. Anderson Cancer Center). TOPRO-3 was obtained from Molecular Probes (Eugene, OR, USA). Antibodies specific for $\beta$-tubulin $(\mathrm{H}-235)$ and $\mathrm{p}$ ERK (E-4) were purchased from Santa Cruz Biotechnology (Santa Cruz, CA, USA). The IRDye 800CW EGF targeting agent, as well as the Odyssey Infrared Imaging and Aerius Automated Infrared Imaging Systems, were provided by LICOR Biosciences (Lincoln, NE, USA).

\section{Characterization of IRDye 800CW EGF binding specificity in vitro}

The binding specificity of the IRDye $800 \mathrm{CW}$ EGF compound was evaluated on A431 and PC3M-LN4 cells by an In-Cell Western assay [23] and [24]. Briefly, cells were grown to approximately $90 \%$ confluency in a microtiter plate (Nunc, Roberts, WI, USA) and starved for $2 \mathrm{~h}$ in serum-free media. For binding assays, starvation media were replaced with media containing increasing concentrations of IRDye $800 \mathrm{CW}$ only $(0.002-3.3 \mu \mathrm{M})$ or IRDye $800 \mathrm{CW}$ EGF $(0.01-70 \mathrm{nM})$. Specificity of the labeled compound was evaluated by competition assays in which starvation media were replaced with media containing unlabeled EGF (in increasing concentrations from 0.01 to $2.14 \mu \mathrm{M})+$ IRDye $800 \mathrm{CW}$ EGF $(70 \mathrm{nM})$ or C225 $(0.5-$ $200 \mu \mathrm{g} / \mathrm{ml})+$ IRDye $800 \mathrm{CW}$ EGF $(70 \mathrm{nM})$. Cells were incubated at room temperature $\left(25^{\circ} \mathrm{C}\right)$ for 2 min and fixed with $4 \%$ formaldehyde solution for $20 \mathrm{~min}$. Four washes in $1 \times$ phosphate-buffered saline (PBS) $+0.1 \%$ Triton X100 were done to remove unbound dye and permeabilize the cells. The plates were blocked in Odyssey Blocking Buffer for $1.5 \mathrm{~h}$ and then incubated with TO-PRO-3 (1:5000) for normalization of cell number using the 700$\mathrm{nm}$ channel. Washing steps were repeated, and the plate was scanned on Aerius. Quantifications were normalized by ratiometric analysis of the $700-\mathrm{nm}$ values applied to the 800 -nm values. 


\section{Preparation of targeting agents}

High-quality targeting agents were prepared by standard fast protein liquid chromatography (FPLC) methods. All separations were performed on an Agilent 1100 series HPLC system (Agilent Technologies, Santa Clara, CA, USA). Sample detection employed a diode array detector set at 260 and $780 \mathrm{~nm}$, and purifications were carried out at $5{ }^{\circ} \mathrm{C}$. Standard FPLC conditions were achieved with this instrument using an isocratic method suitable for size exclusion chromatography. Following conjugation, the coupling mixture was applied to a $35 \times 250$-mm Omnifit column (Western Analytical Products, Wildomar, CA, USA) packed with Superdex 30 gel filtration media (GE Healthcare/Amersham Biosciences, Piscataway, NJ, USA). This column was equilibrated and eluted at $0.5 \mathrm{ml} / \mathrm{min}$ ( 6 bar pressure) with isocratic $1 \times \mathrm{PBS}(\mathrm{pH} 7.4)$. The eluted material was filter sterilized by passage through a $0.2-\mu \mathrm{m}$ syringe filter into a sterile container. Absorbance at $280 \mathrm{~nm}$ was used to calculate EGF concentration from the published molar extinction coefficient. Dual wavelength absorbances were used to determine the dye/protein ratio, which was maintained consistently across all coupling reactions. Dual wavelength absorbance traces from size exclusion chromatography were also used to determine the relative amounts of coupled and unreacted dye in all preparations.

Poor-quality targeting agents were prepared by subjecting unlabeled EGF or IRDye $800 \mathrm{CW}$ EGF to HPLC purification, which is detrimental to protein activity because of the harsh denaturing solvent conditions [25]. A reversephase C18 Zorbax column $(250 \times 9.6 \mathrm{~mm}, 300 \AA$ pore size, $5 \mu \mathrm{m}$ particle size) was used. Solvents included aqueous 50 $\mathrm{mM}$ triethylammonium acetate (TEAA, $\mathrm{pH}$ 5.9) (A) and acetonitrile (B) in a gradient of $20 \% \mathrm{~B}$ to $31 \% \mathrm{~B}$ in $15 \mathrm{~min}$. The purification was performed at a flow rate of $4.7 \mathrm{ml} /$ min (100 bar pressure). Sample was protected from light, dried overnight under vacuum, reconstituted in $1 \times \mathrm{PBS}$, and applied to a cation $\left(\mathrm{Na}^{+}\right.$resin) exchange column equilibrated with $1 \times$ PBS. Following elution from the column with $1 \times$ PBS, the sample was lyophilized. Protein concentration, dye/protein ratio, and unreacted dye quantity were measured as above.

\section{Quantification of downstream signaling by IRDye 800CW $E G F$}

The ability of the targeting agent to stimulate ERK phosphorylation in A431 cells was compared with that of unlabeled EGF. Cells at 90\% confluence in a 96-well plate were serum starved for $2 \mathrm{~h}$. Starvation media were replaced with stimulation media containing 0.03 to $14 \mathrm{nM}$ IRDye $800 \mathrm{CW}$ EGF (FPLC), IRDye 800CW EGF (HPLC), unlabeled EGF (FPLC), or unlabeled EGF (HPLC). Cells were incubated for $2 \mathrm{~min}$ at room temperature followed by fixation and per- meabilization as above. An initial scan on Aerius was done to obtain the level of signal from the conjugated EGF bound to receptors. Then plates were blocked for $1.5 \mathrm{~h}$ in Odyssey Blocking Buffer followed by incubation with anti- $\beta$-tubulin $(1: 100)$ and anti-p-ERK $(1: 100)$ for $1.5 \mathrm{~h}$ at room temperature. Plates were washed four times in $1 \times \mathrm{PBS}+0.1 \%$ Tween 20 prior to incubation with IRDye $800 \mathrm{CW}$ goat antirabbit (1:800) and IRDye 680 goat anti-mouse (1:200) antibody conjugates for $1 \mathrm{~h}$. Cells were washed again, and the plate was scanned on the Aerius. The final ratiometric analysis was adjusted for the initial binding signal, and p-ERK signal was normalized to $\beta$-tubulin.

\section{Subcutaneous injections}

Male NOD/SCID mice (Jackson Laboratory, Bar Harbor, $\mathrm{ME}, \mathrm{USA}$ ) and athymic $\mathrm{Nu} / \mathrm{Nu}$ mice (Charles River, Wilmington, MA, USA) used in these experiments were maintained under the supervision and guidelines of the University of Nebraska-Lincoln Institutional Animal Care and Use Committee. PC3M-LN4 cells were trypsinized, washed, and resuspended at $5 \times 10^{6}$ cells $/ \mathrm{ml}$ in serum-free MEM [26]. Each NOD/SCID mouse was injected subcutaneously in the flank with $100 \mu \mathrm{l}$ of cell suspension $\left(0.5 \times 10^{6}\right.$ cells $)$ using a $1-\mathrm{ml}$ insulin syringe. Tumors reached approximately $0.5 \mathrm{~cm}$ in size at 4 weeks. A431 cells were trypsinized, washed, and resuspended at $2 \times 10^{7}$ cells $/ \mathrm{ml}$ in serum-free medium for subcutaneous injections in the flank $\left(2 \times 10^{6}\right.$ cells $)$ of male athymic $\mathrm{Nu} / \mathrm{Nu}$ mice. Animals were carried for approximately 2 weeks until tumors reached roughly $0.5 \mathrm{~cm}$ in size.

\section{In vivo imaging of IRDye 800CW EGF}

NIR fluorescence imaging in live animals was performed with a research prototype LI-COR Biosciences small animal imager. The LI-COR instrument is a light tight chamber equipped with a cooled charge-coupled device (CCD) camera, area illumination via diode lasers, and a selection of excitation and detection optical fluorescent filters tuned specifically for IRDye 800CW. Images were acquired and analyzed with Wasabi software (Hamamatsu Photonics, Hamamatsu City, Japan) or Adobe Photoshop (Adobe Systems, San Jose, CA, USA). NOD/SCID mice were shaved prior to image collection. All mice were anesthetized with $2 \%$ isoflurane throughout procedures.

To establish initial conditions, IRDye 800CW EGF ( $1 \mathrm{nmol}$ ) was injected via the tail vein into a tumor-negative mouse of each strain and evaluated for systemic clearance by NIR imaging at intervals of 1 to $24 \mathrm{~h}$ over a period of 3 days, after which time more than $90 \%$ of the signal had cleared. Then mice bearing subcutaneous PC3M-LN4 or A431 tumors (diameter $\sim 0.5 \mathrm{~cm}$ ) were injected with IRDye $800 \mathrm{CW}$ EGF (1 nmol) and evaluated for tumor-specific retention of the agent by NIR imaging at 24-h intervals for up to 8 days. 
Signal-to-noise ratios (SNRs) calculated as described below were used to determine an optimal time point of 72 to $96 \mathrm{~h}$ for imaging tumors. Subsequently, an additional eight athymic mice with A431 tumors were injected to compare signal strength of the targeting agents prepared by different methods. One mouse was injected with $0.9 \%$ saline, one mouse received unconjugated IRDye $800 \mathrm{CW}$, two mice were given IRDye $800 \mathrm{CW}$ EGF (1 nmol) from sources A and B purified by FPLC, and four mice were injected with HPLC-damaged IRDye $800 \mathrm{CW}$ EGF $(1 \mathrm{nmol})$ purified by HPLC. Animals were imaged 3 days postinjection and euthanized. Tumors were excised, weighed, measured, and paraffin embedded for section scan analysis on Odyssey.

\section{Statistical analysis}

Images for each mouse were normalized using the same intensity scale with a common minimum and maximum value. SNR was calculated using the following formula:

$$
\mathrm{SNR}=\frac{[(\text { mean intensity tumor })-(\text { mean intensity background })]}{\text { standard deviation of mean background }}
$$

Regions of interest (ROIs) with identical areas were used for both tumor and background. ROIs were quantified for total pixel and mean pixel values. The standard deviation of mean backgrounds was calculated using three to five ROIs. This calculation yields the number of standard deviations over background represented by the suspect tumor/signal. Due to tumor size differences between the animals receiving quality targeting agents, tumor signal minus background signal of similar-size ROI was then corrected for area (pixels). The percentage difference from FPLC-purified agent was determined for HPLC-purified agents.

\section{Tissue section analysis}

After final imaging (day 3 or 4 postinjection), animals were sacrificed. Tumors were excised, scanned on Odyssey, weighed, measured, and preserved in Z-fix (Anatech, Battle Creek, MI, USA), followed by paraffin embedding and tissue sectioning. Sections (5 $\mu \mathrm{m}$ thickness) were scanned in both the 700-nm channel, for tissue autofluorescence, and the 800$\mathrm{nm}$ channel, for the targeted IRDye $800 \mathrm{CW}$ EGF fluorescence signal using Odyssey. The area-weighted fluorescence signal from the 800-nm channel was used to compare targeting agent specificities among experimental conditions.

\section{Results and discussion}

\section{In vitro specificity of IRDye $800 C W$ EGF for tumor cell lines}

Effective use of whole animal NIR optical imaging for sensitive detection of tumor tissue requires highly specific biomarkers that can be labeled to illuminate tumor cells within live mice. Maximal emission of IRDye $800 \mathrm{CW}$ is $789 \mathrm{~nm}$, so it falls in an optimal region of the spectrum to obtain superior signal strength. We previously coupled EGF to IRDye $800 \mathrm{CW}$ and found this targeting agent to be highly sensitive and specific due to its strong signal [23]. Our goal in the current study was to determine an aspect of EGF biological function quantifiable in vitro that reflects maximal tumor targeting potential.

We first characterized binding of the targeting agent to A431 cells by a microplate NIR fluorescence assay as we had done previously for PC3M-LN4 human prostate tumor cells [23]. Confluent monolayer cultures of A431 cells were established in 96-well plates and treated with increasing concentrations of IRDye $800 \mathrm{CW}$ EGF to establish a doseresponse curve for binding of the targeting agent to EGF receptors on the cells. Fluorescence signal became significant at $1 \mathrm{nM}$ concentrations and increased exponentially in a concentration-dependent fashion (Figure 1A). To verify receptor targeting, we performed the same measurements with unconjugated dye, showing negligible increases in fluorescence over a similar concentration course (Figure 1A, circles). The specificity of the conjugate for the EGF receptor on the cells was evaluated by competitions with unlabeled EGF (Figure 1B) and with C225, a monoclonal antibody that blocks ligand binding by the EGF receptor [27] (Figure 1C). Unlabeled EGF reduced fluorescence in a concentration-dependent manner with an $\mathrm{IC}_{50}$ of approximately $0.1 \mu \mathrm{M}$. The antibody competition was also dose dependent with an $\mathrm{IC}_{50}$ of approximately $10 \mu \mathrm{g} / \mathrm{ml}$. These results are comparable to those obtained with prostate tumor cells. Importantly, saturation of binding to whole cells occurred above $0.1 \mu \mathrm{M}$. The value was approximately $0.16 \mu \mathrm{M}$ for PC3M-LN4 cells, showing a half-maximal binding constant of approximately 70 to $80 \mathrm{nM}$, in good agreement with the inhibition constant for unlabeled EGF. Thus, coupling of EGF to the fluorophore did not affect receptor affinity.

To confirm that bioactivity of the targeting agent was indicated accurately by binding affinity, we used an In-Cell Western assay that was developed previously for the quantification of kinase phosphorylation in whole cells [24]. No significant decrease in ERK phosphorylation was noted between cells treated with equimolar concentrations of unlabeled EGF and IRDye 800CW EGF (data not shown). This is consistent with results from other investigators who have shown that both fluorescein isothiocyanate (FITC)-EGF [7] and fluorescent quantum dot-EGF [28] conjugates are effectively internalized by ligand-induced endocytosis of the bound EGF receptor complex, also a downstream effect of biologically intact EGF.

\section{Impairment of binding of EGF and EGF-stimulated ERK phosphorylation by HPLC purification}

We next evaluated whether the microplate binding and In-Cell Western assays could effectively distinguish between 

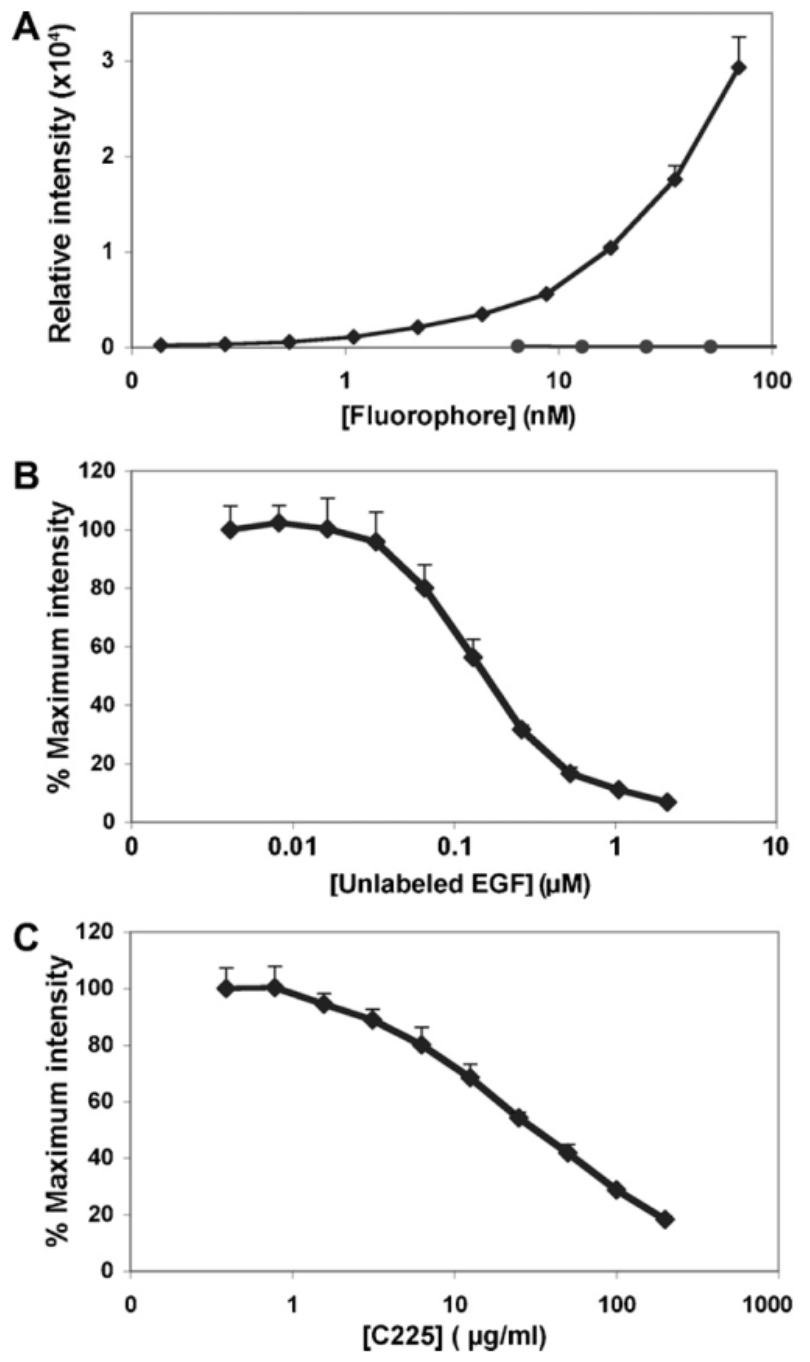

Figure 1. In vitro binding specificity of IRDye $800 \mathrm{CW}$ EGF for tumor cells in culture. A431 cells cultured to $90 \%$ confluence in 96well plates were incubated with increasing concentrations of IRDye 800CW EGF (diamonds) or unconjugated nonreactive IRDye 800CW (circles) for $2 \mathrm{~min}$ and then washed, fixed, permeabilized, and stained with TO-PRO-3 (A). For competitions, cells were incubated with a fixed concentration of IRDye $800 \mathrm{CW}$ EGF $(70 \mathrm{nM})$ containing the indicated concentrations of unlabeled EGF (B) or C225, an EGF receptor blocking antibody (C). All plates were scanned at two wavelengths on the Aerius near-infrared scanner. The 800 -nm signal, normalized to the $700-\mathrm{nm}$ control, is plotted as the means \pm SD of three replicate wells.

ligands with differential binding affinities. To obtain low-affinity ligands for comparisons, we conjugated IRDye $800 \mathrm{CW}$ to EGF from four independent commercial sources and subjected each conjugate to HPLC purification. Binding of these four targeting agents (denoted A, B, C, and D in Figure 2) to A431 cells was compared by NIR fluorescence intensity to the binding of standard maximally active FPLC-purified conjugate from sources A and B. All four preparations demonstrated significant reductions in binding (up to $60 \%$ ) at all concentrations (Figure 2A). Thus, the damage inflicted on EGF
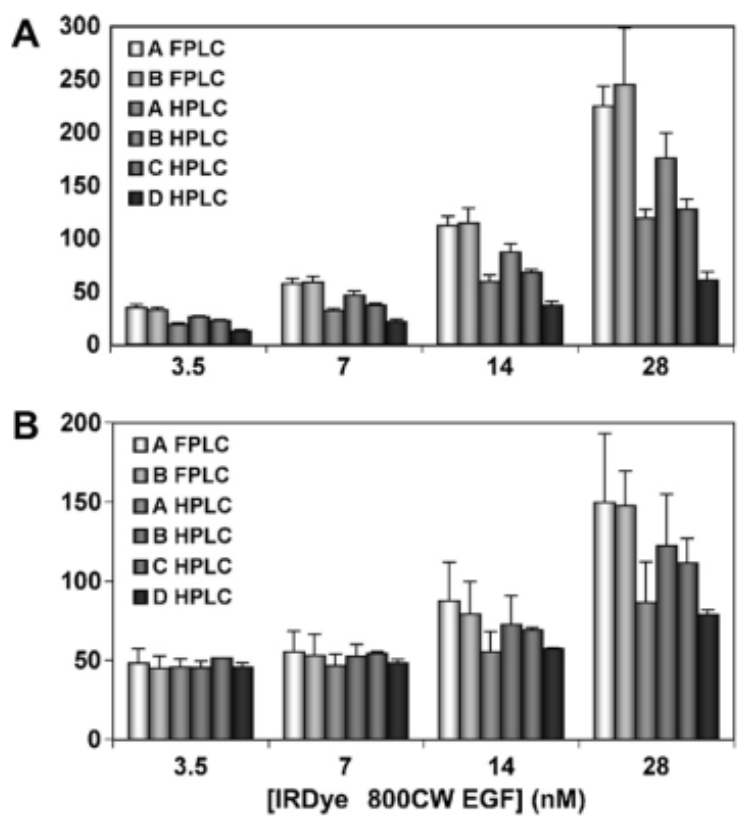

Figure 2. Biological activity elicited by labeled EGF. EGF from four sources (denoted as A, B, C, and D) was conjugated to IRDye $800 \mathrm{CW}$ and purified by HPLC. A431 cells at $90 \%$ confluence in $96-$ well plates were stimulated with the indicated concentrations of the respective agents or with the maximally active FPLC-purified conjugates corresponding to sources $\mathrm{A}$ and $\mathrm{B}$. The plate was scanned to quantify binding of the IRDye $800 \mathrm{CW}$ EGF targeting agents (A). The extent of ERK phosphorylation stimulated in each condition was assessed in the same plate by the In-Cell Western assay using antibodies to total ERK and p-ERK (B). The mean \pm SD for three replicate wells is plotted.

by HPLC treatment dramatically affected its cell binding potential. When we assayed p-ERK levels using the four targeting agents compared with unlabeled (data not shown) or conjugated EGF from sources A and B (Figure 2B), the best performance of any HPLC-purified compound was $28 \%$ of unlabeled EGF stimulation. Reduced levels of ERK phosphorylation confirmed compromised function of the HPLCtreated targeting agents.

\section{Kinetics of IRDye 800CW EGF clearance in vivo}

Ultimately, our goal was to determine whether impaired function of the EGF receptor targeting agent in vitro would correlate with poor tumor targeting in mice. We first determined clearance kinetics of the targeting agent in normal and tumor-bearing mice. Clearance was monitored by injecting animals with $1 \mathrm{nmol}$ of FPLC-purified IRDye $800 \mathrm{CW}$ EGF via the tail vein and collecting NIR images over time from $15 \mathrm{~min}$ to $24 \mathrm{~h}$ (Figure 3A). The pseudo-colored images depict the color-enhanced fluorescence intensity superimposed on white light images of a representative mouse. Fluorescence intensity in regions of interest encompassing the whole animal (large ROI) or limited to the abdominal area (small ROI) was quantified to evaluate clearance. We 
A
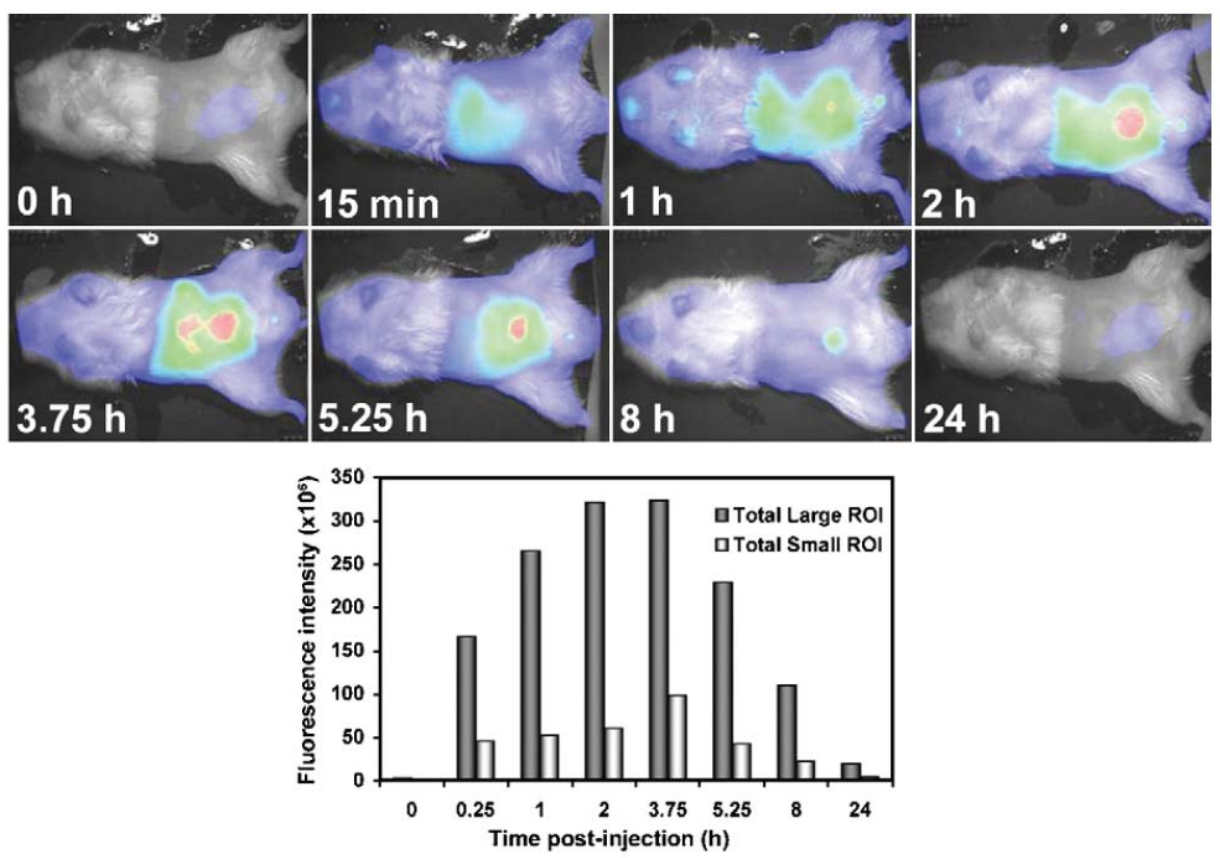

B
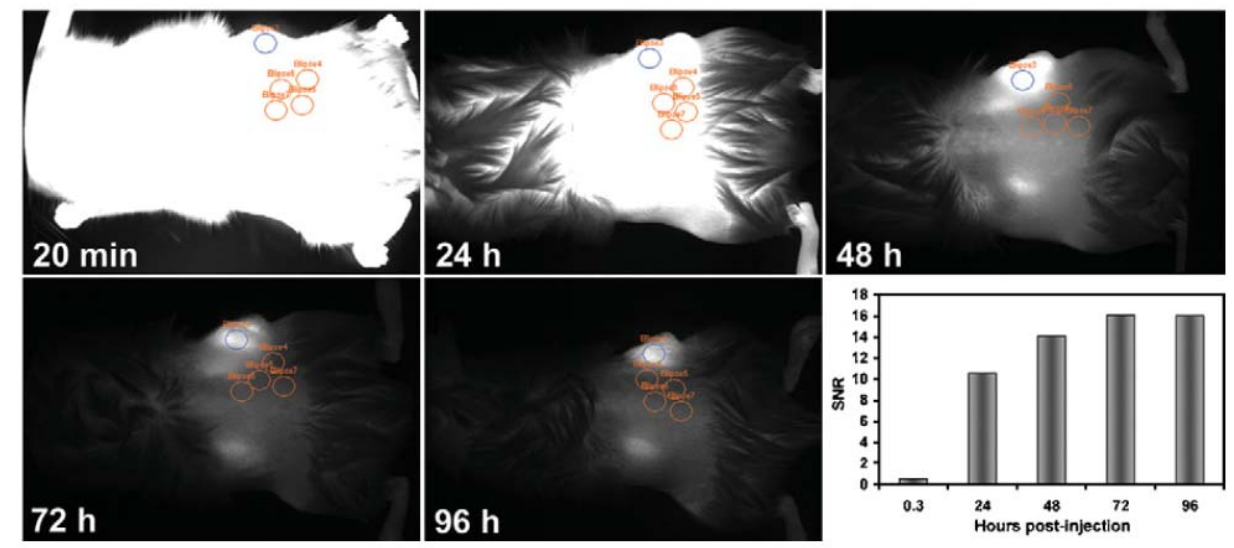

Figure 3. Clearance kinetics of IRDye $800 \mathrm{CW}$ EGF from normal and tumor-bearing mice. (A) IRDye $800 \mathrm{CW}$ EGF (1 nmol) was injected intravenously into a normal NOD/SCID mouse. Images were collected prior to injection $(0 \mathrm{~h})$ and at the indicated times postinjection. Results are shown as color-enhanced fluorescence intensity superimposed on the white light images in the ventral view. Signal intensities (at 789 nm) were normalized to background fluorescence and plotted using the whole animal (large ROI, dark bars) or an arbitrary abdominal ellipse (small ROI, light bars) as ROIs. (B) IRDye $800 \mathrm{CW}$ EGF was injected intravenously into mice with subcutaneous PC3M-LN4 prostate tumor xenografts, and images were collected at the indicated times postinjection. SNR was calculated using a single elliptical ROI denoted on the tumor in blue and four background ellipses (orange) placed arbitrarily in irrelevant adjacent sites on the flank as shown. Fluorescence is also present in the kidneys in these dorsal views. Calculated SNR was plotted to obtain an optimal imaging time point for subsequent experiments.

observed an initial increase in whole animal fluorescence intensity for the first 3 to $4 \mathrm{~h}$ as the targeting agent circulated to the capillaries of the skin. Subsequently, the signal was seen to pass through the liver, intestines, kidneys, and bladder. The analysis confirmed that $75 \%$ of the signal cleared after $8 \mathrm{~h}$ and that more than $90 \%$ cleared after $24 \mathrm{~h}$.

Then we monitored a similar time course for clearance of the targeting agent from tumor-positive animals (Figure 3B). Mice bearing subcutaneous tumors of approximately $0.5 \mathrm{~cm}$ in diameter (measured by digital calipers) were injected as above with IRDye 800CW EGF, imaged immediately, and imaged repeatedly at 24-h intervals. As observed for the tu- mor-negative controls, whole animal and abdominal signal intensity peaked at 3 to $4 \mathrm{~h}$ but had diminished by more than $90 \%$ within $24 \mathrm{~h}$ (data not shown). Analysis of SNR comparing mean fluorescence intensity in an arbitrarily chosen ROI within the tumor, designated as a blue ellipse, to the mean of four background areas of identical size, shown as orange ellipses, exhibited an initial time-dependent increase. Eventual saturation of SNR indicated that the time of maximal sensitivity for imaging was 72 to $96 \mathrm{~h}$, and subsequent images were collected at $72 \mathrm{~h}$ (Figure 3B). In vivo, IRDye 800CW EGF fluorescence following systemic clearance was amplified in the tumors relative to the whole animals and was also specific, 


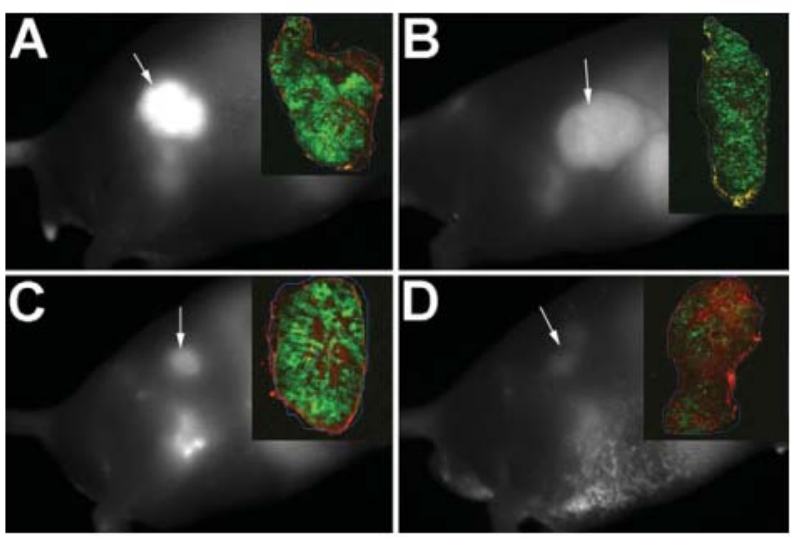

Figure 4. Targeting agent quality analysis in vivo. Male athymic $\mathrm{Nu} /$ Nu mice bearing A431 subcutaneous tumors ( $~ 0.5 \mathrm{~cm}$ diameter) were injected intravenously with IRDye 800CW EGF (1 nmol) purified by FPLC (A and C) or HPLC (B and D). Panels A and B illustrate use of the conjugate to EGF from source A in Figure 3, and for panels $\mathrm{C}$ and D source B was used. Images were collected on anesthetized animals at $72 \mathrm{~h}$ postinjection. Tumors were excised, fixed, paraffin embedded, and sectioned for fluorescence signal determination by twocolor scanning on the Odyssey (inset panels).

as shown by antibody blocking. The sensitivity of this fluorescence signal lends support to the choice of EGF receptor as an epithelial or prostate tumor target and demonstrates that IRDye $800 \mathrm{CW}$ is an effective labeling fluorophore.

\section{Subcutaneous tumor targeting by IRDye 800CW EGF}

To optimize targeting and tumor-specific signal accumulation in noninvasive imaging, it is important to understand the functional parameters of the biomarker and its response to the target. Using the In-Cell Western assay, we detected impaired binding and response of the EGF receptor signaling cascade when the EGF conjugate was subjected to HPLC treatment. We performed an animal imaging study to determine whether impaired binding function would translate to poor tumor signal. FPLC- or HPLC-purified IRDye $800 \mathrm{CW}$ EGF from two sources (A and B) was injected via the tail vein into athymic mice bearing approximately 0.5 $\mathrm{cm}$ subcutaneous A431 tumors. Mice were imaged 3 days postinjection (Figure 4). The arrowheads in Figure 4 indicate positions of detected tumor tissue in these representative fluorescence images, which have been corrected for background. Figs. 4A and $\mathrm{C}$ illustrate signal intensity in animals injected with the FPLC-purified targeting agent, and Figs. 4B and D show the effect of the corresponding HPLC form. These images were analyzed to determine total fluorescence in the tumor (Table 1). Mice receiving HPLC-purified targeting agents from the two different preparations had approximately 28 and $64 \%$ lower signal compared with mice injected with minimally damaged agents (Table 1). Similar results were obtained when damaged agents were injected in mice bearing $22 \mathrm{Rv} 1$ prostate tumors, confirming that this
Table 1. Summary of tumor imaging results

\begin{tabular}{|c|c|c|c|c|c|}
\hline \multicolumn{2}{|c|}{$\begin{array}{l}\text { IRDye } 800 \mathrm{CW} \\
\text { EGF }\end{array}$} & \multicolumn{2}{|c|}{$\begin{array}{l}\text { Normalized } \\
\text { fluorescence }\end{array}$} & \multicolumn{2}{|l|}{ Signal loss } \\
\hline Preparation & Source & $\begin{array}{l}\text { Intact } \\
\text { animals }^{\mathrm{a}}\end{array}$ & $\begin{array}{l}\text { Tumor } \\
\text { sections }^{\mathrm{b}}\end{array}$ & $\begin{array}{l}\text { Intact } \\
\text { animals (\%) }\end{array}$ & $\begin{array}{l}\text { Tumor } \\
\text { sections (\%) }\end{array}$ \\
\hline FPLC & A & 1039.30 & 94.23 & & \\
\hline FPLC & B & 512.77 & 113.39 & & \\
\hline HPLC & A & 746.22 & 69.58 & $28^{\mathrm{c}}$ & 26 \\
\hline HPLC & B & 185.94 & 55.92 & $(64)^{d}$ & (51) \\
\hline HPLC & $\mathrm{C}$ & 100.21 & 59.44 & $90(80)$ & $37(48)$ \\
\hline HPLC & $\mathrm{D}$ & 85.63 & 55.75 & $92(83)$ & $41(51)$ \\
\hline
\end{tabular}

${ }^{a}$ Normalized fluorescence for intact animals: mean pixel fluorescence (tumor) minus mean pixel fluorescence (background).

b $800 \mathrm{~nm}$ fluorescence per area $\left(\mathrm{mm}^{2}\right)$.

${ }^{\mathrm{c}}$ Comparison with source A FPLC to determine percentage loss.

${ }^{\mathrm{d}}$ Comparison with source B FPLC to determine percentage loss (numbers in parentheses).

phenomenon is not unique to the A431 cells. In images not shown, additional mice were injected with HPLC-purified probes from sources $\mathrm{C}$ and $\mathrm{D}$, but the fluorescence signal from these animals was diminished 80 to $90 \%$ with respect to that calculated for the animals receiving optimized preparations (Table 1). Thus, the magnitude of the differences varied considerably, but a targeting agent exhibiting suboptimal signaling potential in vitro correlated strongly with poor signal strength in live animal imaging.

Finally, tumors were excised and sectioned for fluorescence scanning analysis to verify whether signal intensity measured in the animal was attributable to fluorescence in the tumor tissue and whether the intensity was consistent throughout the tumor. Sections were scanned on the Odyssey in two channels, and the corresponding merged images are shown as insets in Figure 4. Red represents tissue autofluorescence in the 700-nm channel, and green denotes specific fluorescence in the 800-nm channel resulting from IRDye 800CW EGF. Normalized fluorescence of the tissue sections is presented in Table 1. Signal intensity was evident throughout the tumors irrespective of targeting agent. Consistent with results from intact animal image analysis, sections exhibited signal reductions of approximately 25 to $60 \%$. Thus, performance of targeting agents damaged by HPLC purification, as identified in vitro by the assay for ERK phosphorylation, again translated to poor outcomes in whole animal imaging experiments.

In conclusion, we have characterized an effective targeting agent for noninvasive optical detection and tracking of tumors in mice. The fluorophore used, IRDye $800 \mathrm{CW}$, can be easily coupled to many ligand or targeting molecules of interest. We have found that an in vitro assay that detects binding and efficacy of EGF-conjugated products in stimulating downstream biological function is an accurate estimate of targeting efficacy in vivo. We expect this application to have further value in probe and drug development as a correlate assay for other biolabels to estimate competitive ligand and/or drug interactions in vivo. 


\section{Acknowledgments}

The authors acknowledge Rose Skopp for excellent technical assistance and Joe Barycki and Mike Olive for critical evaluation of the manuscript.

\section{References}

[1] J.V. Frangioni, In vivo near-infrared fluorescence imaging, Curr. Opin. Chem. Biol. 7 (2003), pp. 626-634.

[2] V. Ntziachristos, J. Ripoll, L.V. Wang and R. Weissleder, Looking and listening to light: the evolution of whole-body photonic imaging, Nat. Biotechnol. 23 (2005), pp. 313-320.

[3] G. Choy, P. Choyke and S.K. Libutti, Current advances in molecular imaging: noninvasive in vivo bioluminescent and fluorescent optical imaging in cancer research, Mol. Imaging 2 (2003), pp. 303-312.

[4] T. Troy, D. Jekic-McMullen, L. Sambucetti and B. Rice, Quantitative comparison of the sensitivity of detection of fluorescent and bioluminescent reporters in animal models, Mol. Imaging 3 (2004), pp. 9-23.

[5] H.W. Kang, R. Weissleder and A. Bogdanov Jr., Targeting of MPEG-protected polyamino acid carrier to human E-selectin in vitro, Amino Acids 23 (2002), pp. 301-308.

[6] K. Licha, N. Debus, S. Emig-Vollmer, B. Hofmann, M. Hasbach, D. Stibenz, S. Sydow, M. Schirner, B. Ebert, D. Petzelt, C. Buhrer, W. Semmler and R. Tauber, Optical molecular imaging of lymph nodes using a targeted vascular contrast agent, $J$. Biomed. Opt. 10 (2005), p. 41205.

[7] K.L. Carraway III and R.A. Cerione, Fluorescent-labeled growth factor molecules serve as probes for receptor binding and endocytosis, Biochemistry 32 (1993), pp. 12039-12045.

[8] S. Ke, X. Wen, M. Gurfinkel, C. Charnsangavej, S. Wallace, E.M. Sevick-Muraca and C. Li, Near-infrared optical imaging of epidermal growth factor receptor in breast cancer xenografts, Cancer Res. 63 (2003), pp. 7870-7875.

[9] D. Citrin, A.K. Lee, T. Scott, M. Sproull, C. Menard, P.J. Tofilon and $\mathrm{K}$. Camphausen, In vivo tumor imaging in mice with nearinfrared labeled endostatin, Mol. Cancer Ther. 3 (2004), pp. 481-488.

[10] A. Becker, C. Hessenius, K. Licha, B. Ebert, U. Sukowski, W. Semmler, B. Wiedenmann and C. Grotzinger, Receptor-targeted optical imaging of tumors with near-infrared fluorescent ligands, Nat. Biotechnol. 19 (2001), pp. 327-331.

[11] F.F. Jobsis, Noninvasive, infrared monitoring of cerebral and myocardial oxygen sufficiency and circulatory parameters, Science 198 (1977), pp. 1264-1267.

[12] B. Chance, Optical method, Annu. Rev. Biophys. Biophys. Chem. 20 (1991), pp. 1-28.

[13] O.H. Aina, J. Marik, R. Gandour-Edwards and K.S. Lam, Nearinfrared optical imaging of ovarian cancer xenografts with novel $\alpha_{3}$-integrin binding peptide "OA02", Mol. Imaging 4 (2005), pp. 439-447.

[14] X. Chen, P.S. Conti and R.A. Moats, In vivo near-infrared fluorescence imaging of integrin $\alpha_{\mathrm{v}} \beta_{3}$ in brain tumor xenografts, Cancer Res. 64 (2004), pp. 8009-8014.
[15] Z. Cheng, Y. Wu, Z. Xiong, S.S. Gambhir and X. Chen, Nearinfrared fluorescent RGD peptides for optical imaging of integrin $\alpha_{\mathrm{v}} \beta_{3}$ expression in living mice, Bioconj. Chem. 16 (2005), pp. 1433-1441.

[16] W. Wang, S. Ke, Q. Wu, C. Charnsangavej, M. Gurfinkel, J.G. Gelovani, J.L. Abbruzzese, E.M. Sevick-Muraca and C. Li, Near-infrared optical imaging of integrin $\alpha_{\mathrm{v}} \beta_{3}$ in human tumor xenografts, Mol. Imaging 3 (2004), pp. 343-351.

[17] M.H. Kraus, N.C. Popescu, S.C. Amsbaugh and C.R. King, Overexpression of the EGF receptor-related proto-oncogene erbB-2 in human mammary tumor cell lines by different molecular mechanisms, EMBO J. 6 (1987), pp. 605-610.

[18] G. Di Lorenzo, G. Tortora, F.P. D’Armiento, G. De Rosa, S. Staibano, R. Autorino, M. D'Armiento, M. De Laurentiis, S. De Placido, G. Catalano, A.R. Bianco and F. Ciardiello, Expression of epidermal growth factor receptor correlates with disease relapse and progression to androgen-independence in human prostate cancer, Clin. Cancer Res. 8 (2002), pp. 3438-3444.

[19] A. Levitzki, EGF receptor as a therapeutic target, Lung Cancer 41 (2003) (Suppl. 1), pp. S9-S14.

[20] N.J. Maihle, A.T. Baron, B.A. Barrette, C.H. Boardman, T.A. Christensen, E.M. Cora, J.M. Faupel-Badger, T. Greenwood, S.C. Juneja, J.M. Lafky, H. Lee, J.L. Reiter and K.C. Podratz, EGF/ErbB receptor family in ovarian cancer. In: M.S. Stack and D.A. Fishman, Editors, Ovarian Cancer, Kluwer Academic, Boston, MA (2002), pp. 247-258.

[21] A.P. Kyritsis and H. Saya, Epidemiology, cytogenetics, and molecular biology of brain tumors, Curr. Opin. Oncol. 5 (1993), pp. 474-480.

[22] A. Sorkin and M. Von Zastrow, Signal transduction and endocytosis: close encounters of many kinds, Nat. Rev. Mol. Cell Biol. 3 (2002), pp. 600-614.

[23] J.L. Kovar, M.A. Johnson, W.M. Volcheck, J. Chen and M.A. Simpson, Hyaluronidase expression induces prostate tumor metastasis in an orthotopic mouse model, Am. J. Pathol. 169 (2006), pp. 1415-1426.

[24] H. Chen, J. Kovar, S. Sissons, K. Cox, W. Matter, F. Chadwell, P. Luan, C.J. Vlahos, A. Schutz-Geschwender and D.M. Olive, A cell-based immunocytochemical assay for monitoring kinase signaling pathways and drug efficacy, Anal. Biochem. 338 (2005), pp. 136-142.

[25] N.H.H. Heegaard and C. Schou, Affinity ligands in capillary electrophoresis. In: D.S. Hage, Editor, Chromatographic Science vol. 92, CRC Press, Boca Raton, FL (2006).

[26] M.A. Simpson, C.M. Wilson and J.B. McCarthy, Inhibition of prostate tumor cell hyaluronan synthesis impairs subcutaneous growth and vascularization in immunocompromised mice, $\mathrm{Am}$. J. Pathol. 161 (2002), pp. 849-857.

[27] J. Harding and B. Burtness, Cetuximab: An epidermal growth factor receptor chemeric human-murine monoclonal antibody, Drugs Today (Barc) 41 (2005), pp. 107-127.

[28] D.S. Lidke, P. Nagy, R. Heintzmann, D.J. Arndt-Jovin, J.N. Post, H.E. Grecco, E.A. Jares-Erijman and T.M. Jovin, Quantum dot ligands provide new insights into erbB/HER receptor-mediated signal transduction, Nat. Biotechnol. 22 (2004), pp. 198-203. 